placebo in combination with adjuvant chemotherapy with/without radiotherapy in patients with EC.

Methods Eligible patients are $\geq 18$ years with newly diagnosed high-risk (stage I/II non-endometrioid or with p53 abnormality and any histology, stage III/IVa), previously untreated EC following surgery with curative intent with no evidence of disease post-operatively. $\square 990$ patients will be randomized to receive pembrolizumab $200 \mathrm{mg}$ or placebo Q3W for 6 cycles plus chemotherapy (carboplatin area under the curve [AUC] 5/ 6 plus paclitaxel $175 \mathrm{mg} / \mathrm{m}^{2} \mathrm{Q} 3 \mathrm{~W}$ or carboplatin AUC 2/2.7 plus paclitaxel $\left.60 \mathrm{mg} / \mathrm{m}^{2} \mathrm{QW}\right)$ in stage 1 . Patients receive pembrolizumab $400 \mathrm{mg}$ or placebo Q6W for 6 cycles in stage 2. Radiotherapy (external beam radiotherapy [EBRT] and/or brachytherapy) \pm radiosensitizing cisplatin $50 \mathrm{mg} / \mathrm{m}^{2}$ (days 1 and 29) may be administered after completion of chemotherapy. Randomization is stratified by MMR status (pMMR vs dMMR) and, within pMMR, by planned radiation therapy (cisplatin-EBRT vs EBRT vs no EBRT), histology (endometrioid vs non-endometrioid), and FIGO surgical stage (I/II vs III/IVA). Dual primary endpoints are disease-free survival (DFS; per investigator assessment) and OS. Secondary endpoints include DFS (per BICR), DFS (per investigator assessment) and OS by biomarker status (PD-L1 and tumor mutational burden), safety, and QoL. Enrollment began December 2020 and is ongoing in 28 countries.

Results Not applicable

Conclusions Not applicable

\section{EPV108/\#267 PITFALLS IN PRE-OPERATIVE PREDICTION OF LYMPH NODE METASTASIS IN EARLY ENDOMETRIAL CANCER}

${ }^{1} \mathrm{P}$ Veena*, ${ }^{2} \mathrm{R}$ llango, ${ }^{2} \mathrm{~J}$ Durairaj. ${ }^{1}$ Jawaharlal Institute of Postgraduate Medical Education and Research, Obstetrics and Gynecology, Puducherry, India; ${ }^{2}$ IIPMER, Obgyn, Puducherry, India

\subsection{6/ijgc-2021-IGCS.178}

Objectives The role of lymphadenectomy in early-stage endometrial cancer is controversial as it is associated with intraoperative complications, and its therapeutic benefit is not established. Prediction of lymph nodal metastasis to perform selective lymph node dissection is desirable. This study was conducted to study the grade of the tumor obtained by endometrial biopsy specimen and depth of myometrial invasion assessed by imaging pre-operatively as predictors of lymph nodal metastasis in early endometrial cancers.

Methods This was a cross-sectional study where we studied 100 patients from August 2016 to May 2018. After Ethical Committee clearance, all patients diagnosed with early endometrial cancer in our hospital were included in the study after getting informed consent. Pre-operative tumor grade and depth of myometrial invasion were studied as predictors of lymph nodal metastasis.

Results The incidence of positive lymph node metastasis in our study was $18.6 \%$. Both pre-operative tumor grade and depth of myometrial invasion were not significantly associated with lymph node metastasis. There was significant variation between pre-operative and post-operative tumor grade and depth of myometrial invasion. Among postoperative histopathological factors, only lymphovascular space invasion was significantly associated with lymph node metastasis.

Conclusions In our study, neither pre-operative nor postoperative grade of the tumor and depth of myometrial invasion

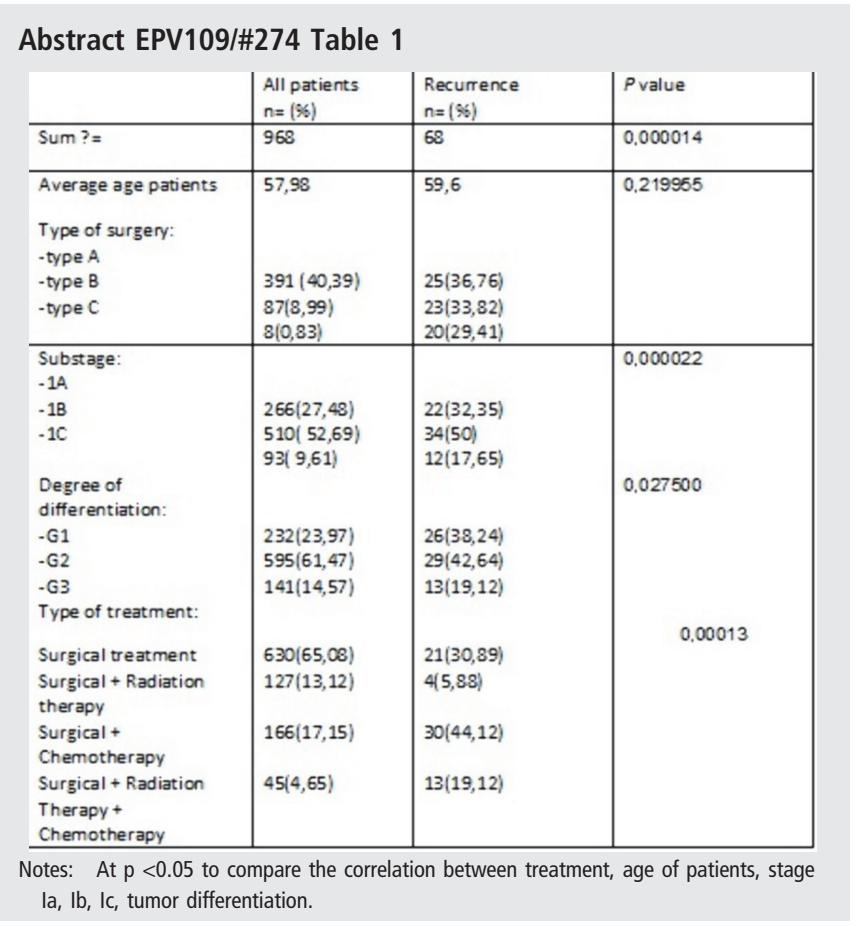

were significantly associated with lymph node metastasis. There was considerable variation between pre-op and post-op grades of the tumor, making pre-op grade an unreliable factor in predicting lymph node metastasis in endometrial cancer. Among postoperative histopathological factors, only lymphovascular space invasion was significantly associated with lymph node metastasis.

\section{EPV109/\#274 ANALYSIS OF THE FREQUENCY OF ENDOMETRIAL CANCER STAGE I}

O Movchan*, v Svintsitskiy, O Renkas. National Cancer Institute, Oncogynecology, Kiyv Ukraine

\subsection{6/ijgc-2021-IGCS.179}

Objectives In 2020, according to the National Cancer Registry, endometrial cancer in the structure of cancer took second place $(10.9 \%)$ after breast cancer. Although the detection and treatment of the disease in the early stages has good prospects, but there are relapses from $2 \%$ to $26 \%$, according to various literature.

Methods The analysis of recurrence rate among 968 patients with endometrial cancer and stages of endometrioid type. Recurrences amounted to 68 cases $(7.02 \%)$. The staging took place according to the 1988 FIGO classification. The following statistical methods were used: standard descriptive, parametric and nonparametric. Differences at $p<0.05$ were considered significant.

Results The analysis was performed depending on the characteristics of the tumor process and the type of treatment, the recurrence rate was estimated - see table 1 . The average age of patients ranged from 25 to 85 years. The recurrence time was detected, on average, after 36 months \pm 15.97 months. In combination treatment, receiving adjuvant radiation therapy, recurrences were most often detected - after 6-18 months \pm 13.53 months. Long-term recurrences were detected after a combination of surgical treatment with chemotherapy at 32- 
64 months \pm 14.31 months. Table 1 Comparison of recurrence rates depending on the characteristics of the tumor process and the type of treatment

Conclusions Our study demonstrates that the frequency of their occurrence is approximately the same, regardless of the method of treatment used. However, with relapses, life expectancy is significantly reduced.

\section{EPV110/\#289 ENDOMETRIAL ASPIRATION FOR ENDOMETRIAL CANCER DIAGNOSIS}

L Barbosa*, B Napoleao, M Ribeiro, M Pagani, V Silveira, R Grande, F Campos. Universidade do Vale do Sapucai, Oncoginecologia, Pouso Alegre, Brazil

10.1136/ijgc-2021-IGCS. 180

Objectives The diagnosis of endometrial cancer, the third most common among gynecological tumors in Brazil, must be made by anatomopathological examination of the biopsy of the endometrial cavity using hysteroscopy or semiotic curettage. Recently, the endometrial aspirate technique has been used in order to speed up the diagnosis as it is an easy, low cost, outpatient method, dispensing with more complex tests, such as hysteroscopy. The aim of the study was to compare the results of this technique with those of semiotic uterine curettage in women with suspected endometrial hyperplasia/carcinoma.

Methods Analytical and retrospective study by analyzing the medical records of 52 women between 41 and 83 years old at the outpatient clinic of Hospital das Clínicas Samuel Libânio, Brazil. Material collected by means of endometrial aspirate and uterine curettage from patients with endometrial thickening on ultrasound, with or without bleeding, uterine bleeding after menopause or abnormal uterine bleeding.

Results 52 patients evaluated with endometrial aspirate, 12 were diagnosed with endometrial adenocarcinoma and three with hyperplasia with endometrial atypia. The endometrial aspirate was positive in 8 of the adenocarcinomas, suspected in two and negative in two other cases. In atypical hyperplasia, aspirate was positive in one case and negative in two.

Conclusions The use of endometrial aspirate for diagnosis was $66.6 \%$ positive in this study, a satisfactory method in scenarios of limited availability of more accurate tests. However, further studies are needed to assess the sensitivity/specificity of the method, as well as standardization in the collection and interpretation of the findings.

\section{EPV111/\#303 SENTINEL LYMPH NODE MAPPING FOR ENDOMETRIAL CANCER: A PROSPECTIVE STUDY ABOUT THIRTY EIGHT CASES}

${ }^{1} S$ Sghaier*, ${ }^{2} \mathrm{H}$ Bouaziz, ${ }^{3} \mathrm{~N}$ Tounsi, ${ }^{2} \mathrm{M}$ Bouhani, ${ }^{4} \mathrm{~L}$ Zaabar, ${ }^{5} \mathrm{G}$ Sahraoui, ${ }^{2} \mathrm{H}$ Bouzainne, ${ }^{2} \mathrm{M}$ Slimane, ${ }^{2} \mathrm{~K}$ Rahal. ${ }^{1}$ Salah Azaiez Institute, Surgical Oncology Department, Tunis, Tunisia; ${ }^{2}$ Salah Azaiez Institute of Oncology, Surgical Oncology, Tunis, Tunisia; ${ }^{3}$ salah Azaiz Institut, Department of Surgical Oncologists, Tunis, Tunisia; ${ }^{4}$ Salah Azaiez Institute, Nuclear Medicine, Tunis, Tunisia; ${ }^{5}$ Salah Azaiez Institute, Anatomopathology, Tunis, Tunisia

\subsection{6/ijgc-2021-IGCS.181}

Objectives Endometrial cancer (EC) represents the most common gynecological cancer mostly diagnosed at stage I. Sentinel lymph node (SLN) arised as a valuable option to lymph node dissection. We aim to determine negative predictive value (NPV), overall and bilateral detection rates of SLN in EC stage I.
Methods This was a cross-sectional prospective study including 38 patients with EC stage I treated at Salah Azaiz Institute over a period of 34 months from March 2018 to January 2021.

Results Endometrioid adenocarcinoma was reported in $89 \%$ of cases. The pelvic MRI showed IA and IB stages in 58\% and $42 \%$ of cases, respectively.The detection techniques were combined (48\%), colorimetric (34\%) and radioisotope (18\%). Lymphoscintigraphy was conducted in $66 \%$ of women demonstrating overall, bilateral and failed detection rates of $92 \%$, $24 \%$ and $8 \%$, respectively. The overall, bilateral and failed intra-operative detection rates were of $76 \%, 37 \%$ and $24 \%$ respectively. A micrometastasis (1\%) was noted among a total of 87 SLNs. False negative rate (FNR) and NPV were of $0 \%$ and $100 \%$.Factors affecting overall detection wereinitial histologic grade $(p=0.01)$ and tumor size on MRI $(p=0.04)$. Final histologic grade $1(p=0.005), 2(p=0.002)$ and myometrial invasion $(\mathrm{p}=0.04)$ were also significant contributors. No significant factors affecting bilateral detection were set.

Conclusions FNR and NPV were of $0 \%$ and $100 \%$ similarly to previous results through literature. We aim to continue this promising protocol toward including more patients that may helps us improve our overall and bilateral detection rates.

\section{EPV112/\#318 UTERINE CARCINOSARCOMA FOLLOWING TAMOXIFEN THERAPY FOR BREAST CANCER: A SERIES OF 11 CASES}

K Ben Hamida*, L Naija, M Ghalleb, MA Bouida, R Chargui, K Rahal. Salah Azaiez Institute of Oncology, Surgical Oncology Department, Tunis, Tunisia

\subsection{6/ijgc-2021-IGCS. 182}

Objectives Uterine carcinosarcoma (UC) is rare and carries a poor prognosis. It represents only $1-2 \%$ of uterine cancers and less than $5 \%$ of all uterine malignancies. Although these tumors usually arise de novo, some cases developed under Tamoxifen therapy have been reported. We aim to report our institution's experience.

Methods A retrospective study of 11 women with endometrial carcinosarcoma after breast cancer were treated at Salah Azaiez Institute of Oncology from 2004 to 2014.

Results The mean age of UC diagnosis was 64 years (50-82 years). All patients were given adjuvant hormone therapy by Tamoxifen for breast cancer. The mean duration of Tamoxifen use was 42 months (3-60 months) with a mean cumulative dose of $25709 \mathrm{mg}$ (1800-36500mg). The main presenting symptom of UC was post-menopausal bleeding. Ultrasound showed thickened endometrium in four cases. Endometrial biopsy revealed UC in three cases. Surgery was performed in ten cases. It consisted of total hysterectomy and bilateral oophorectomy in all cases; we performed lymphadenectomy in three cases. Adjuvant chemotherapy and radiotherapy were performed in three cases. The median follow-up after surgery was nine months (1-64 months). One patient developed a peritoneal recurrence five months after surgery. Three women developed bone metastasis from their uterine cancer, and two patients developed liver metastases from their breast cancer.

Conclusions The survival benefits associated with five-year adjuvant Tamoxifen counterbalances the low morbidity and mortality risk associated with endometrial adenocarcinoma development. Things are different with UC and its pejorative 